\title{
Radiation Hardness and Lifetime Studies of Photodiodes for the Optical Readout of the ATLAS Semiconductor Tracker
}

\author{
D.G. Charlton, J.D. Dowell, R.J. Homer, P. Jovanovic, I.R. Kenyon, \\ G. Mahout, H.R. Shaylor, A. Sibley, J.A. Wilson.
}

School of Physics and Astronomy, University of Birmingham, Birmingham. B15 $2 T T, U K$.

\author{
J.H. Bibby, I-M. Gregor ${ }^{\star}$, R.L. Wastie, A.R. Weidberg. \\ Nuclear and Astrophysics Laboratory, University of Oxford, Keble Road, Oxford. \\ OX1 3RH, UK.
}

\begin{abstract}
A large sample (96) of epitaxial Si PIN photodiodes has been irradiated by $\sim 1 \mathrm{MeV}$ neutrons and $24 \mathrm{GeV}$ protons with fluences up to $10^{15}$ equivalent $1 \mathrm{MeV}$ neutrons per $\mathrm{cm}^{2}$ in order to test their suitability for use in the optical readout of the ATLAS semiconductor tracker and pixel detector at the CERN Large Hadron Collider. After an initial reduction of $30 \%$ the responsivity remains constant up to the maximum fluence. The rise and fall times are not significantly affected and remain below 1 ns. Although the dark current increases linearly with increasing neutron fluence, its level remains below $100 \mathrm{nA}$ which is negligible in comparison to the operating photocurrent which is above $100 \mu \mathrm{A}$. Enhanced ageing studies at $60{ }^{\circ} \mathrm{C}$ have also been carried out and no failure has occurred after an equivalent of 360 years of operation.
\end{abstract}

Key words: Epitaxial Si PIN photodiodes; neutron (1 MeV) and proton (24 GeV) irradiation; dark current; responsivity; ageing; lifetime; ATLAS semiconductor tracker; ATLAS pixel detector; LHC

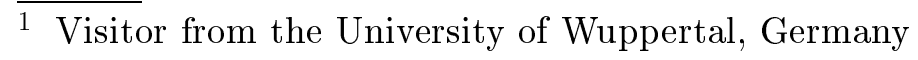




\section{Introduction}

The optical readout [1] of the ATLAS semiconductor tracker (SCT) [2] at the CERN Large Hadron Collider will make use of epitaxial Si PIN photodiodes located on the detector to receive the timing, trigger and control (TTC) signals that initiate and control the readout of binary data at $40 \mathrm{Mbits} / \mathrm{s}$. The 4088 photodiodes required will have to operate for at least ten years in a high radiation environment [3] with estimated integrated fluences of equivalent 1 $\mathrm{MeV}$ neutrons up to $1.8 \times 10^{14} \mathrm{~cm}^{-2}$ and total ionising radiation doses up to $100 \mathrm{kGy}$. Even harsher conditions will exist for the pixel detector [2], which requires a further 2500 photodiodes, with fluences and doses up to $10^{15} \mathrm{n} \mathrm{cm}^{-2}$ and $500 \mathrm{kGy}$ respectively.

In this paper we present the results of comprehensive radiation and lifetime studies carried out on a sample of 96 photodiodes. In an earlier paper [4] we described tests carried out on a smaller sample which showed the photodiodes to be adequately radiation hard. Details of the readout design and basic radiation damage theory are given therein. The damage in silicon results predominantly from the non-ionising energy loss (NIEL) by the incident particles to the semiconductor material. As the damage is found to scale with the NIEL [5-8], it is convenient to express the expected fluences in terms of an effective fluence of a particular particle type at a fixed energy, in this case 1 $\mathrm{MeV}$ neutrons, that would produce the same damage.

The irradiations were carried out with neutrons of around $1 \mathrm{MeV}$ at the Rutherford Appleton Laboratory ISIS facility [11] and with $24 \mathrm{GeV}$ protons at the CERN Proton Synchroton (PS) giving a range of three fluences for

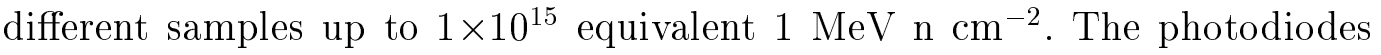
were reverse biassed at $10 \mathrm{~V}$ during both these tests and were read out during the proton test.

Accelerated ageing tests were carried out by operating the 96 photodiodes at an elevated temperature of $60^{\circ} \mathrm{C}$ with a reverse bias of $10 \mathrm{~V}$, and continuously illuminating each photodiode with around $1 \mathrm{~mW}$ of $850 \mathrm{~nm}$ light from a VCSEL (vertical cavity surface emitting laser diode). According to the Arrhenius equation, this gives an accelerated ageing factor $A F$ of

$$
A F=e^{\left(\frac{E_{a}}{k}\right)\left(\frac{1}{T_{1}}-\frac{1}{T_{2}}\right)}
$$

where $E_{a}$ is the activation energy of the devices, $T_{1}$ is the normal operating temperature, $T_{2}$ is the elevated temperature, and $k$ is Boltzmann's constant. 
In the following sections, we describe the structure of the photodiodes and outline the results of the earlier measurements. We then describe the equipment used for the present studies and give some details of the radiation facilities. The results of the present tests are then given followed by the conclusions.

\section{The Photodiodes and Earlier Tests}

The epitaxial silicon PIN photodiodes ${ }^{2}$ (figure $1 \mathrm{~b}$ ) have an active diameter of $350 \mu \mathrm{m}$ and are made from an n-type substrate which has a low-doped background (intrinsic) n-type layer followed by a p-type layer grown epitaxially on top. The low-doped region which forms the depletion layer is only $\sim 15 \mu \mathrm{m}$ thick ${ }^{3}$. The devices can therefore be fully depleted (figure 1a) at relatively low bias voltages $(<10 \mathrm{~V})$ even after radiation damage has increased the carrier concentration.

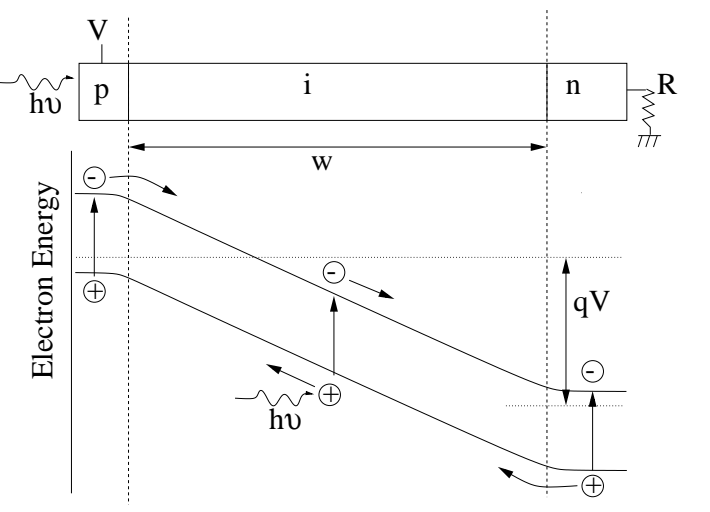

(a)

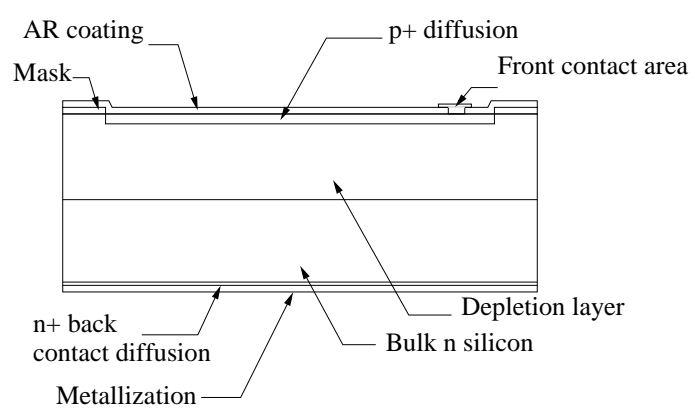

(b)

Fig. 1. Principle a) and section b) of the epitaxial PIN photodiode used for the test.

The irradiated devices also have fast rise and fall times $(<1 \mathrm{~ns})$ as reported in [4]. The responsivity before irradiation is typically $0.5 \mathrm{~A} / \mathrm{W}$ and was found from the earlier measurements [4] to fall to about $70 \%$ of its initial value after a fluence of $\sim 0.25 \times 10^{14} \mathrm{~cm}^{-2}$ equivalent $1 \mathrm{MeV}$ neutrons, remaining at this level after further irradiation up to $4 \times 10^{14} \mathrm{~cm}^{-2}$. On the other hand, the dark

2 Manufactured by Centronic, Croydon, UK.

3 The depeletion layer thickness has to be compared with the mean absorption distance for the light which is $\sim 10 \mu \mathrm{m}$ at $820 \mathrm{~nm}$. 
current rises approximately linearly with increasing fluence reaching $\sim 20 \mathrm{nA}$ at room temperature for a total fluence of $2.5 \times 10^{14} \mathrm{~cm}^{-2}$. The dark current is well known to be temperature sensitive and at $-8{ }^{\circ} \mathrm{C}$ falls to less than $5 \mathrm{nA}$ but continues to rise linearly with increasing fluence. The behaviour, at fixed temperature, can be parameterised by

$$
\Delta I_{d c}=K \phi
$$

where $\Delta I_{d c}$ is the change in the dark current induced by a fluence $\phi$, and $\mathrm{K}$ is known as the damage constant.

The initial response degradation [4] is probably related to type inversion of the low-doped layer from n-type to p-type as a result of the incident radiation forming acceptor levels. However, the detailed mechanism causing the decrease in response is not understood. At low integrated fluence $\left(\phi<10^{13}\right.$ $\mathrm{n} \mathrm{cm}^{-2}$ ), the acceptor state compensates the donor state until the effective doping concentration $N_{e f f}$ is reduced to that of the intrinsic silicon. On the other hand, at higher fluences, the effective doping is mainly provided by the radiation induced defects, and $N_{\text {eff }}$ may be parametrized as [9]:

$$
\left|N_{e f f}\right| \sim \beta \phi
$$

where $\beta$ is a factor related to the rate of creation of an acceptor state per unit path length in silicon.

\section{Experimental Equipment}

The photodiodes are mounted in arrays of eight on ceramic tiles ${ }^{4}$ with a spacing of $2 \mathrm{~mm}$ (see figure 2a). Electrical connections are made via copper pads with the photodiodes wire-bonded to a common anode pad to which the DC bias is applied (see figure 2b). Twelve tiles containing a total of 96 photodiodes were irradiated and three tiles were left in the unirradiated state.

$\overline{4}$ Packaged by Marconi Materials Technology, Caswell, UK. 


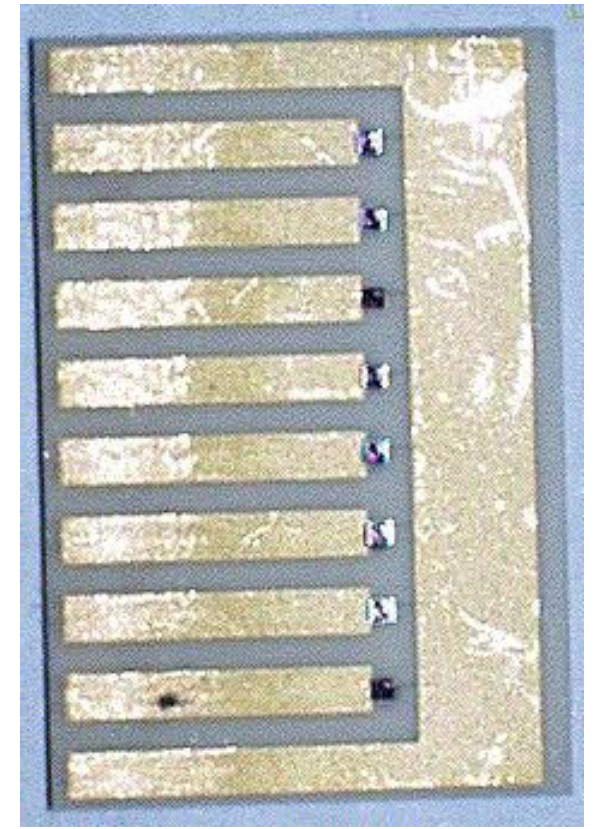

(a)

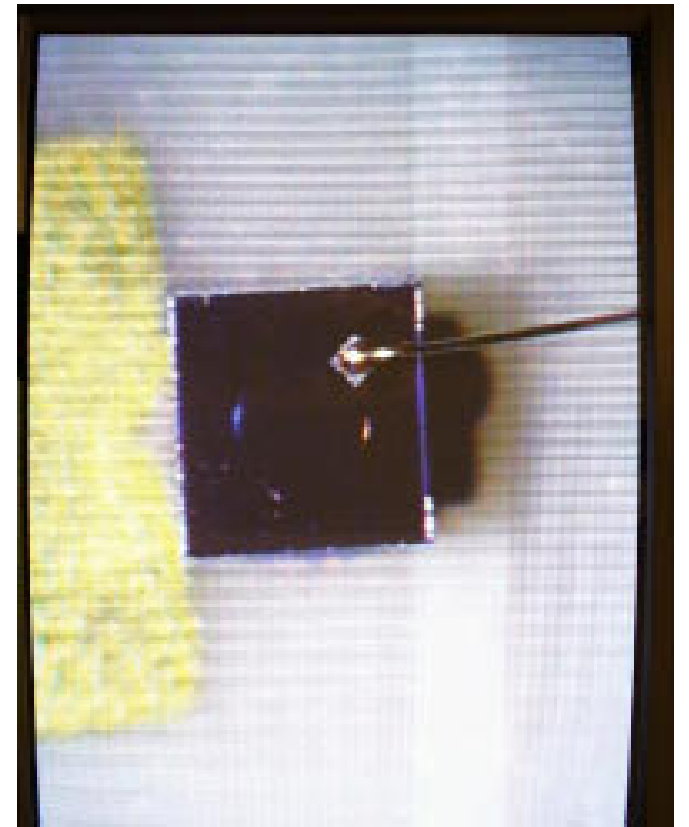

(b)

Fig. 2. a) Eight photodiodes mounted on a ceramic tile, and b) a single photodiode showing the wire bond to the common anode pad.

\subsection{Scanning Machine}

A scanning machine [10] originally built at the University of Berne, Switzerland to characterise large samples of light emitting diodes (LEDs) and VCSELs was adapted to make equivalent measurements on the photodiodes.

The readout scheme is illustrated in figure 3. A single VCSEL light source is scanned over the arrays of photodiodes in fine steps of $0.1 \mathrm{~mm}$ longitudinally and $0.04 \mathrm{~mm}$ transversely. The position giving the maximum signal is found for each photodiode and the dark current and DC response are measured. The photodiodes are mounted on temperature controlled blocks and point downwards. A precise mechanical structure ensures a well defined vertical distance between the VCSEL and the photodiodes and the measurements are reproducible to about $2 \%$.

Further details about the scanning machine and its data recording system can be found in [10].

\subsection{Lifetime Apparatus}

The accelerated ageing tests rely on operating the photodiodes at an elevated temperature. Twelve tiles are precision mounted in metal blocks on a platform 


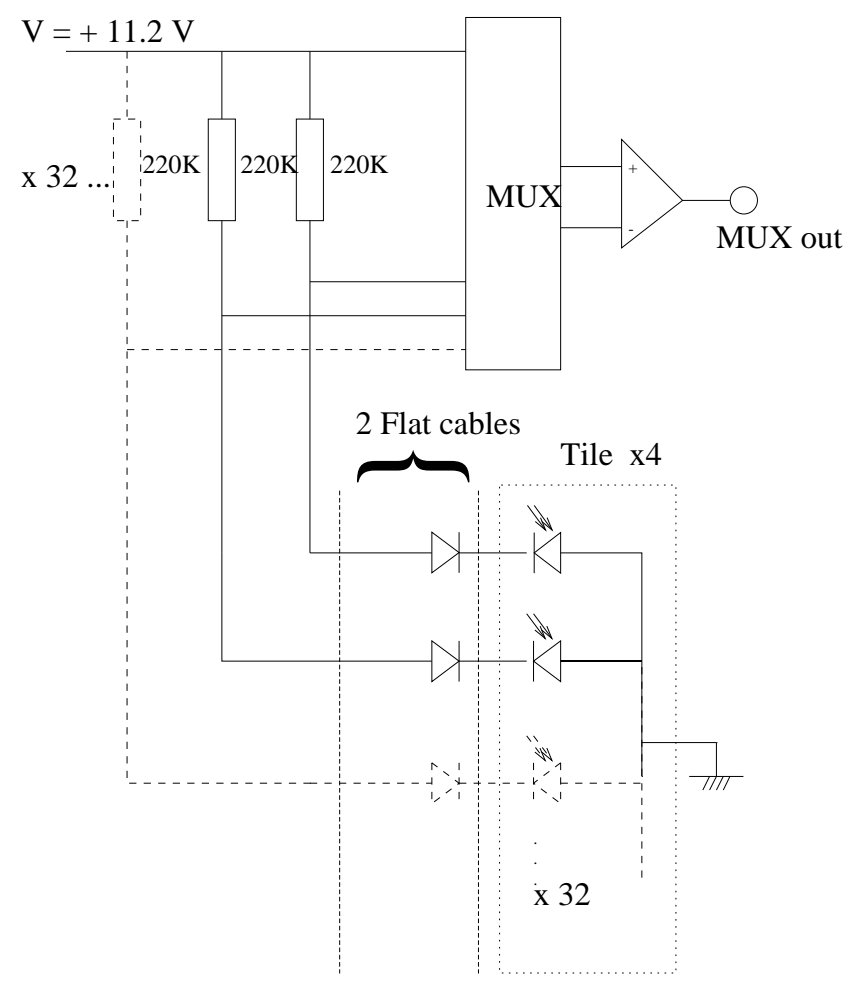

Fig. 3. Readout of the PIN photodiodes in the scanning machine.

that can be heated by resistors to around $60^{\circ} \mathrm{C}$. Each photodiode is illuminated from a separate VCSEL via an optical fibre with a core diameter of 62.5 $\mu \mathrm{m}$ and length of about $30 \mathrm{~cm}$. Figure 4 shows a detail of the equipment used for the lifetime studies.

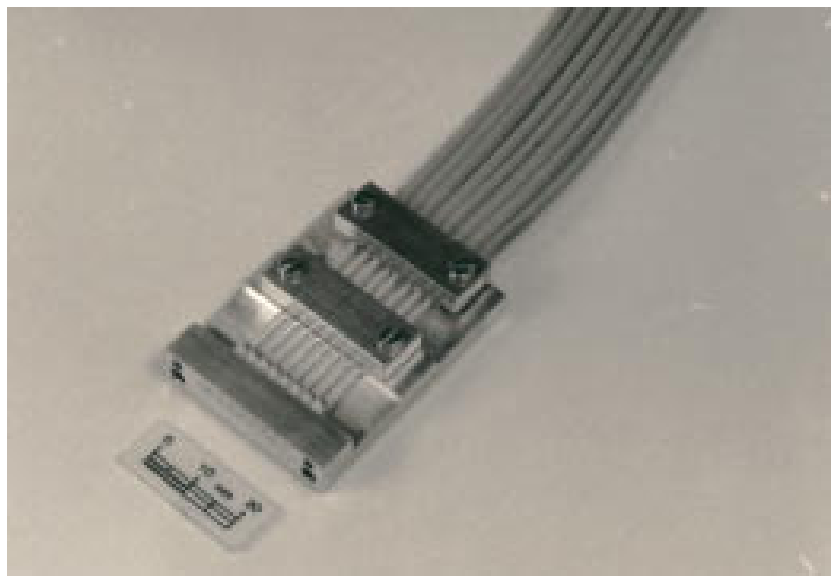

Fig. 4. Fibre mounting block in the ageing apparatus. Each photodiode is illuminated by a VCSEL.

The fibres are glued into metal blocks in sets of eight, accurately spaced to match the spacing of the photodiodes, and the ends are polished. Each of these blocks is screw mounted onto a corresponding tile block. An adjustable voltage source is connected to the tiles to allow a bias to be applied. The 
whole apparatus is enclosed in a metal case which also acts as an interlocked safety shield. Temperature control is achieved by comparing the output of a thermocouple with a reference voltage. The maximum temperature excursion from the nominal set value was about $5{ }^{\circ} \mathrm{C}$.

The nominal current in the VCSELs was $10 \mathrm{~mA}$, giving a received optical power of around $1 \mathrm{~mW}$, and could be set with a precision of $10 \mu \mathrm{A}$. The photocurrent from the photodiodes was typically $300-400 \mu \mathrm{A}$ and could be measured with a precision of $1 \mu \mathrm{A}$.

\section{Irradiation Facilities}

\subsection{ISIS facility}

The neutron irradiations were carried out using the ISIS spallation source at the Rutherford Appleton Laboratory [11]. A similar energy spectrum to that encountered in the ATLAS environment is obtained using neutrons emitted from the collector. This latter is a graphite block backed with copper, designed to stop the $800 \mathrm{MeV}$ protons in the synchrotron not trapped by the RF during the acceleration cycle. A special support permits three different fluxes to be obtained. For example, at the nearest position to the collector and over a cycle of 37 days, a total fluence of $7.5 \times 10^{14} \mathrm{n} \mathrm{cm}^{-2}$ was achieved. The rate is 14 times higher than the $2 \times 10^{7} \mathrm{n} \mathrm{cm}^{-2} \mathrm{~S}^{-1}$ equivalent $1 \mathrm{MeV}$ flux in the pixel detector. The received exposure of the irradiated devices is monitored using cobalt foils with an error of about $20 \%$.

\subsection{CERN Proton Synchrotron}

The energy of the protons from the CERN PS is $24 \mathrm{GeV}$ and fluences of $10^{14}$ $\mathrm{cm}^{-2}$ day $^{-1}$ are obtainable. However, the devices under test were mounted on an $\mathrm{x}-\mathrm{y}$ stage which scans an area $6 \mathrm{~cm} \times 6 \mathrm{~cm}$ perpendicular to the beam direction (designed for the irradiation of silicon detectors). This provides uniform radiation over the area but the fluences are reduced to the order of $10^{13}$ $\mathrm{cm}^{-2}$ day $^{-1}$.

For the proton tests, aluminium foils were used for an initial calibration run. The fluences obtained, via the reaction ${ }^{27} \mathrm{Al}(\mathrm{p}, 3 \mathrm{pn}){ }^{24} \mathrm{Na}$ (producing a 1.37 $\mathrm{MeV}$ gamma ray with a $\sim 15 \mathrm{hr}$ half-life), were used to calibrate a secondary emission counter. The errors on the fluence measurements were of the order of $10 \%$. 


\subsection{The irradiations and their equivalent $1 \mathrm{MeV}$ neutron fluences}

Three subsets of the tiles were subjected to different fluences and one set was kept unirradiated as a reference. A total of 12 tiles were irradiated at ISIS to $1.2 \times 10^{14} \mathrm{n} \mathrm{cm}^{-2}$. Of these, three were then further irradiated with protons at CERN to $2.8 \times 10^{14} \mathrm{p} \mathrm{cm}^{-2}$ and three were given a further $7.5 \times 10^{14} \mathrm{n} \mathrm{cm}^{-2}$ at ISIS. The irradiations and their $1 \mathrm{MeV}$ neutron equivalents are given in Table 1 together with the cumulative fluences for each sample.

Table 1

Details of irradiations at ISIS and CERN, their equivalent $1 \mathrm{MeV}$ neutron fluences, and cumulative fluences for each sample.

\begin{tabular}{|l|c|c|}
\hline Irradiation $\mathrm{cm}^{-2}$ & $1 \mathrm{MeV}$ neutron equivalent $\mathrm{cm}^{-2}$ & Cumulative fluence $\mathrm{cm}^{-2}$ \\
\hline $1.2 \times 10^{14}$ (ISIS) & $1.5 \times 10^{14}$ & $1.5 \times 10^{14}$ \\
$2.8 \times 10^{14}$ (CERN) & $1.5 \times 10^{14}$ & $3.0 \times 10^{14}$ \\
$7.5 \times 10^{14}$ (ISIS) & $9 \times 10^{14}$ & $1.05 \times 10^{15}$ \\
\hline
\end{tabular}

\section{Results of Radiation Studies}

In the following, results are given for three increasing equivalent $1 \mathrm{MeV}$ neutron fluences as well as for the unirradiated sample. A minimum of 24 photodiodes have been been irradiated at each level.

\subsection{Dark current}

The dark current has been measured at room temperature $\left(21^{\circ} \mathrm{C}\right)$ using the scanning machine with a reverse bias of $10 \mathrm{~V}$. The dark current as a function of fluence is shown in figure 5 and exhibits the expected linear behaviour. Using equation 2 , the $\mathrm{K}$ factor is found to be $0.58 \pm 0.02 \times 10^{-13} \mathrm{nA} \mathrm{cm}^{-2}$. A direct comparison of the $\mathrm{K}$ factor with that obtained in the earlier measurements [4] is difficult because in that case the factor quoted was calculated during the irradiation. The factor was twice as big because the effect of annealing was not included. The dark current recorded in one of the photodiodes during the proton irradiation is shown in figure 6 and the annealing effects are clearly visible. The different peaks correspond to the scanning cycles of the beam irradiating the photodiode. Between two peaks, we can observe a decrease of the dark current by a factor two or more. However, with a total dark current of less than $100 \mathrm{nA}$ and a typical operating signal of more than $100 \mu \mathrm{A}$, this 
noise will not affect the performance of the PIN diodes. The lower flux of particles at the LHC will result in an even lower dark current.

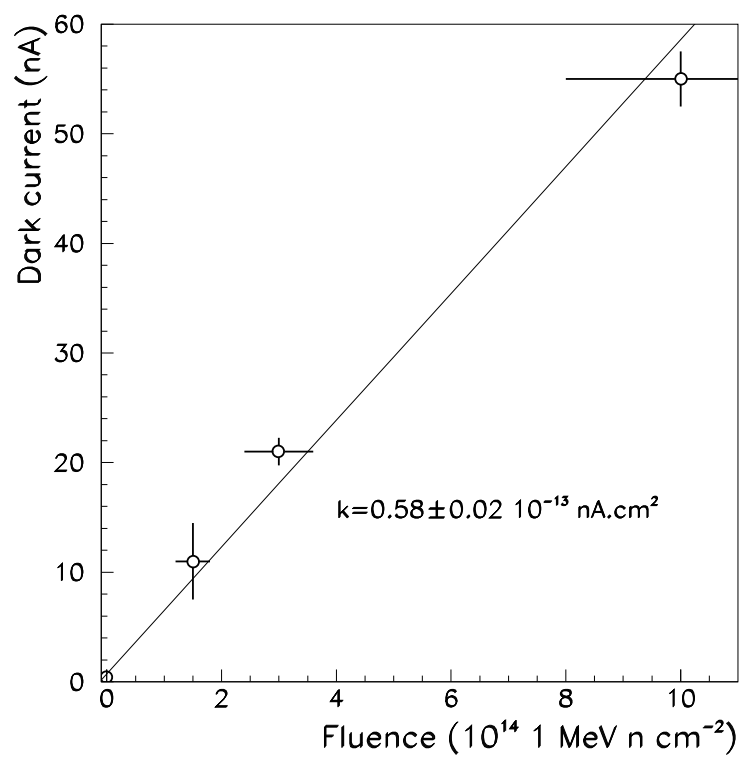

Fig. 5. Behaviour of room temperature dark current as a function of neutron fluence.

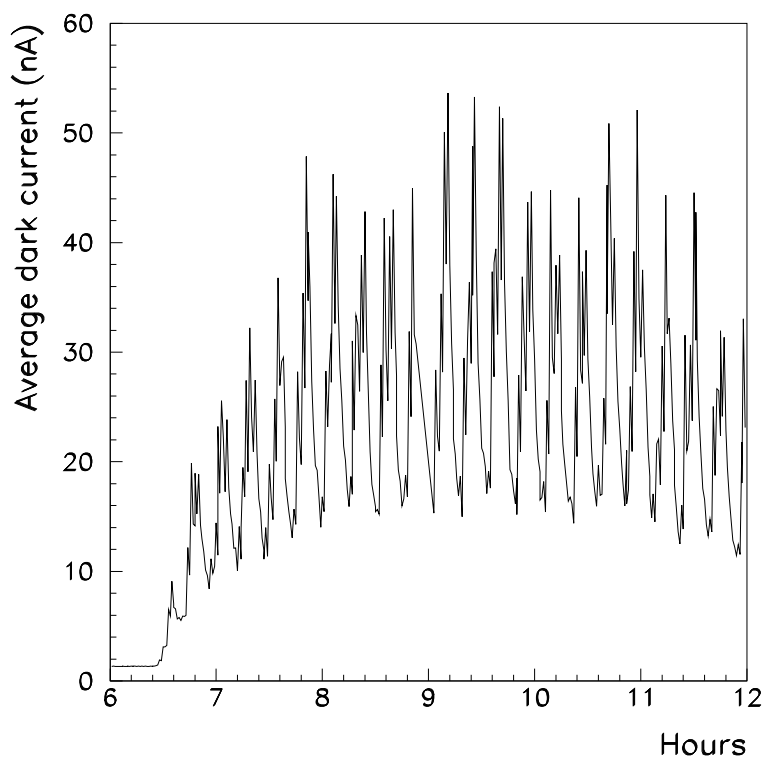

Fig. 6. Behaviour of the dark current in one of the photodiodes as a function of time during the proton irradiation. 


\subsection{Responsivity}

The results for the responsivity before and after irradiation are summarised in Table 2. The distributions of the individual measurements are shown in figure 7. There is an initial decrease to about $70 \%$ of the unirradiated value followed by constant behaviour as the radiation is increased. The results confirm those of [4] but now extend to three times the fluence. Two of the PIN diodes had their bonds broken and are not shown in the figure.

Table 2

Total fluences and average responsivities for the unirradiated and irradiated samples.

\begin{tabular}{|l|c|}
\hline Total Fluence $(1 \mathrm{MeV}) \mathrm{n} \mathrm{cm}^{2}$ & Responsivity $(\mathrm{A} / \mathrm{W})$ \\
\hline Unirradiated & $0.48 \pm 0.01$ \\
$1.5 \times 10^{14}$ & $0.34 \pm 0.01$ \\
$3 \times 10^{14}$ & $0.34 \pm 0.01$ \\
$1 \times 10^{15}$ & $0.32 \pm 0.01$ \\
\hline
\end{tabular}

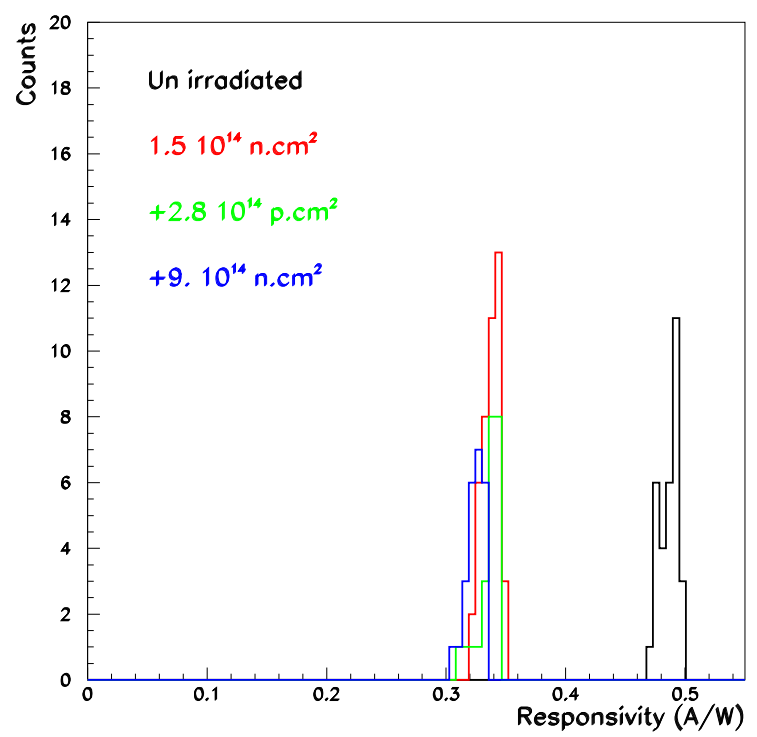

Fig. 7. Individual responsivity measurements for different integrated fluences.

The responsivity as a function of bias voltage for different integrated fluences is shown in figure 8 . The reverse bias voltage required to achieve full depletion increases with increasing fluence and reaches a value of about $8 \mathrm{~V}$ for a fluence of $10^{15} \mathrm{n} \mathrm{cm}^{-2}$.

As discussed in section 1, radiation damage induces type inversion and increases the concentration of p-type carriers. Therefore to fully deplete the PIN 


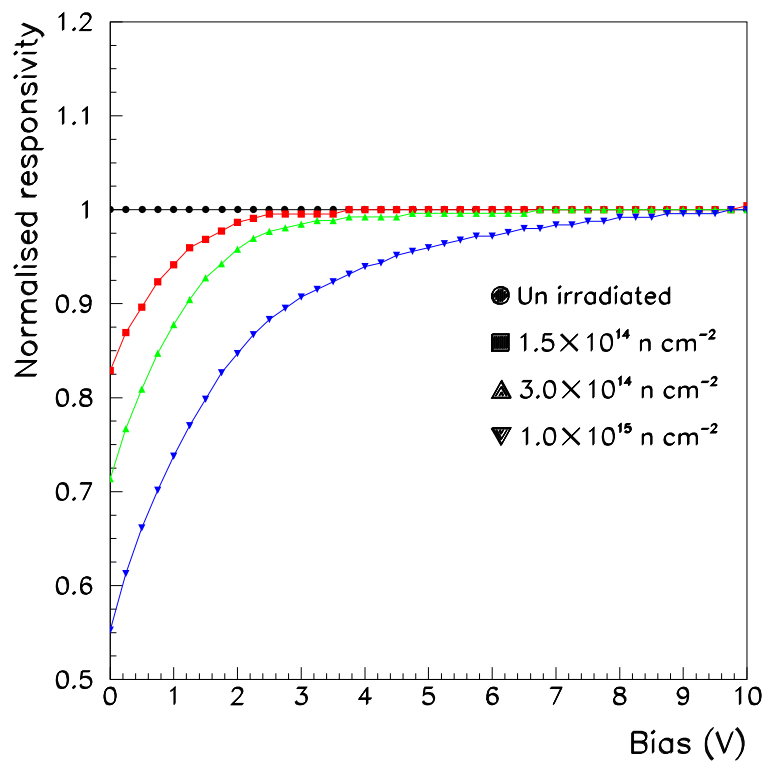

Fig. 8. Normalised responsivity as a function of the bias voltage for different integrated fluences.

diode, we have to increase the bias. From equation 3 , the effective minority carrier concentration $N_{e f f}$ is proportional to the fluence and $N_{\text {eff }}$ determines the external bias voltage $V_{\text {bias }}$ necessary to achieve full depletion:

$$
V_{\text {bias }}+V_{b i}=\frac{e\left|N_{\text {eff }}\right| w^{2}}{2 \epsilon}
$$

with $V_{b i}$ the built-in diffusion potential $\left(V_{b i} \sim 0.65 \mathrm{~V}\right), w$ the $15 \mu \mathrm{m}$ thickness of the depletion layer and $\epsilon$ the dielectric constant of silicon. The bias voltages required to fully deplete the PIN diodes exposed to different fluences can be determined from the onsets of the plateaux of the curves shown in figure 8 . These values can be used with equation 4 to determine the doping concentration $N_{\text {eff }}$ for each fluence and the results are shown in figure 9. We observe the expected linear behaviour, with a constant $\beta=0.039 \mathrm{~cm}^{-1}$. This value is consistent with measurements reported in [12] for silicon detectors.

The temperature dependence of the responsivity, measured using the lifetime apparatus, is shown in figure 10 , and is around $0.25 \%$ per ${ }^{\circ} \mathrm{C}$, similar to the value quoted by the manufacturer but showing some difference between the irradiated and unirradiated samples. 


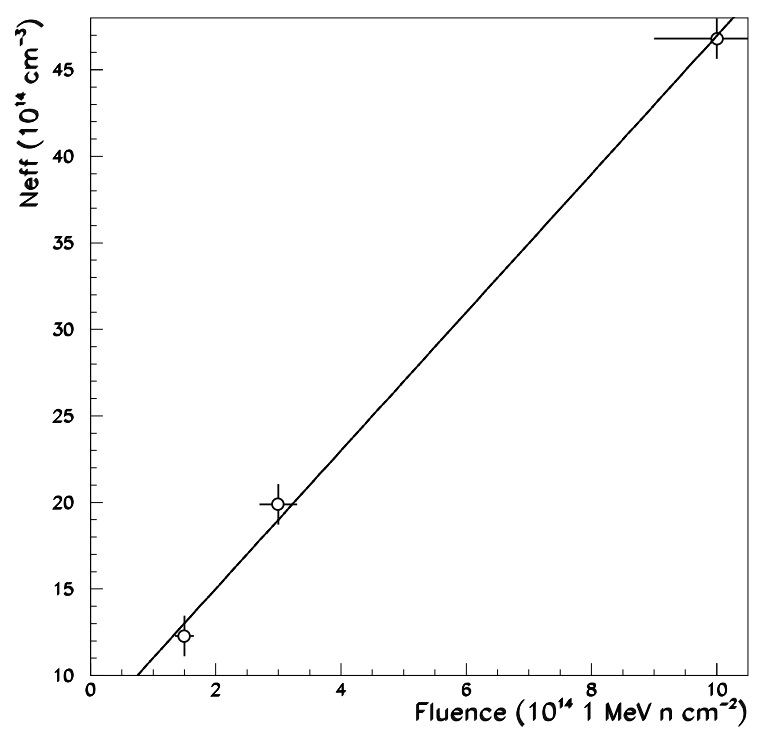

Fig. 9. Effective minority carrier concentration as a function of the integrated fluence.

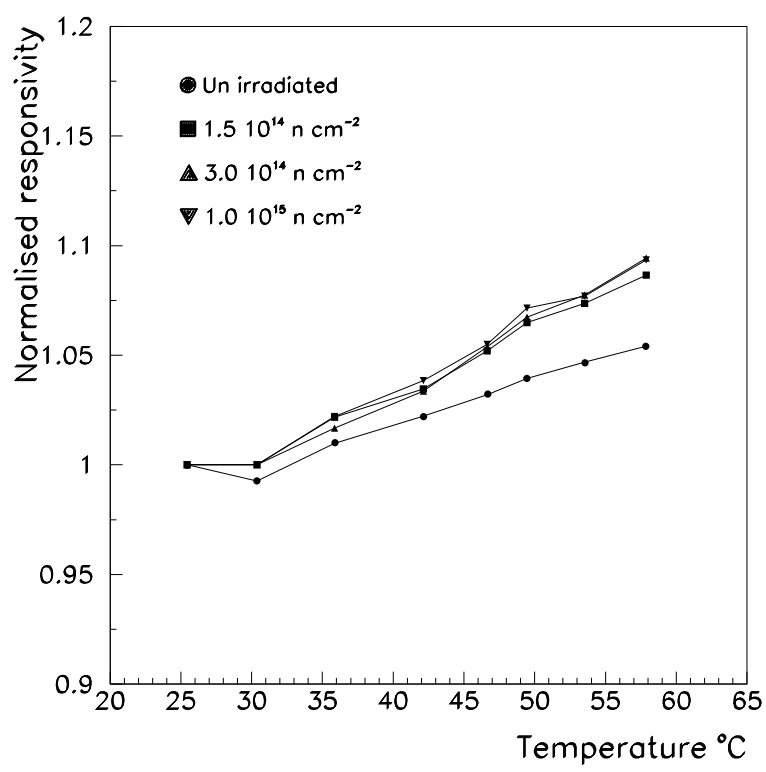

Fig. 10. Behaviour of the responsivity as a function of temperature.

\subsection{Rise time}

The rise and fall times were measured using VCSEL light of typically $1 \mathrm{~mW}$ power through an optical fibre. The test was performed using the lifetime apparatus. At low rise or fall times, the measurements are limited by the speed of the input optical signal and the oscilloscope used to about 1 ns. The 
results for the rise time are plotted in figure 11. Despite the radiation damage, rise and fall times of less than $1 \mathrm{~ns}$ are readily achievable for a bias of $5 \mathrm{~V}$, which is acceptable for this application.

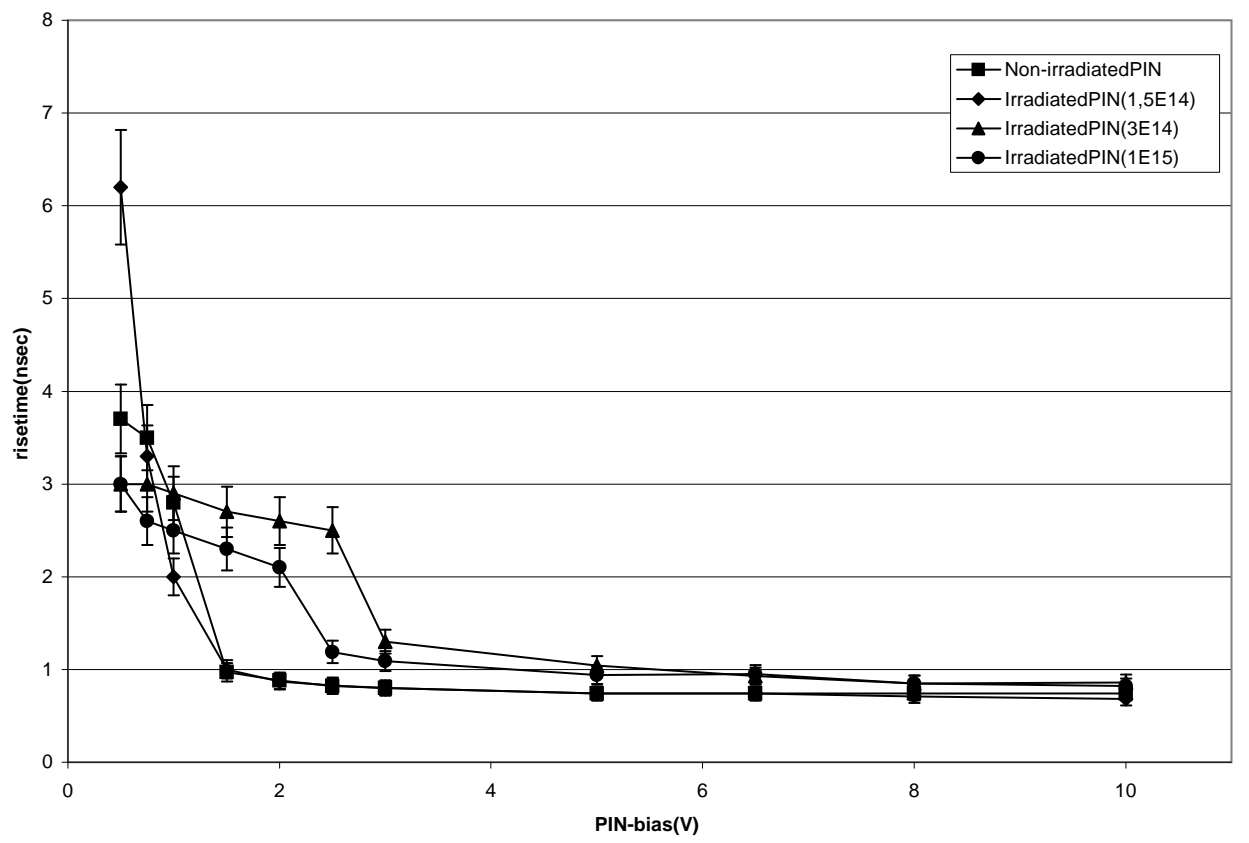

Fig. 11. Rise time as a function of the PIN diode bias voltage for different integrated fluences.

\subsection{Ageing test}

In order to estimate the reliability of the photodiodes, four sets of 24 (see table 3 ), including one unirradiated and three irradiated samples, were illuminated continuously over an extended period and their output measured at regular intervals to detect failures. Assuming that the devices age only when biassed, the operational duty cycle at the LHC ( $\sim 100$ days per year) gives a factor 3 enhancement of the effective lifetime. Further enhancement is provided by operating the photodiodes at an elevated temperature which gives an accelerated ageing factor $A F$ described by equation 1 .

Reliability models define the Mean Time To Failure $(M T T F)$ as the average time that would be expected to elapse before a unit fails. In principle it can be calculated by testing $\mathrm{n}$ components until they all fail and measuring the time $t_{i}$ taken for each device to fail, in which case:

$$
\text { MTTF }=\frac{1}{n} \sum_{i=1} t_{i}
$$


The mean failure rate $\lambda$ and the $M T T F$ are the inverse of one another:

$$
\lambda=\frac{1}{M T T F}
$$

At two different temperatures, using equation 1, we then have the relation:

$$
A F=\frac{\operatorname{MTTF}\left(T_{1}\right)}{\operatorname{MTTF}\left(T_{2}\right)}
$$

The value of $A F$ depends strongly on $E_{a}$, the activation energy. This latter depends upon the type of failure taking place and since different types of failure may be predominant at different points in the lifetime of the device, no well-defined value exists. Furthermore, as seen in figure 12, it is not possible to deduce a value of $E_{a}$ from our data as no diode has failed. The best (conservative) estimate of the activation energy $E_{a}$ was found in [13] (and references cited therein) with a value of $0.6 \mathrm{eV}$.

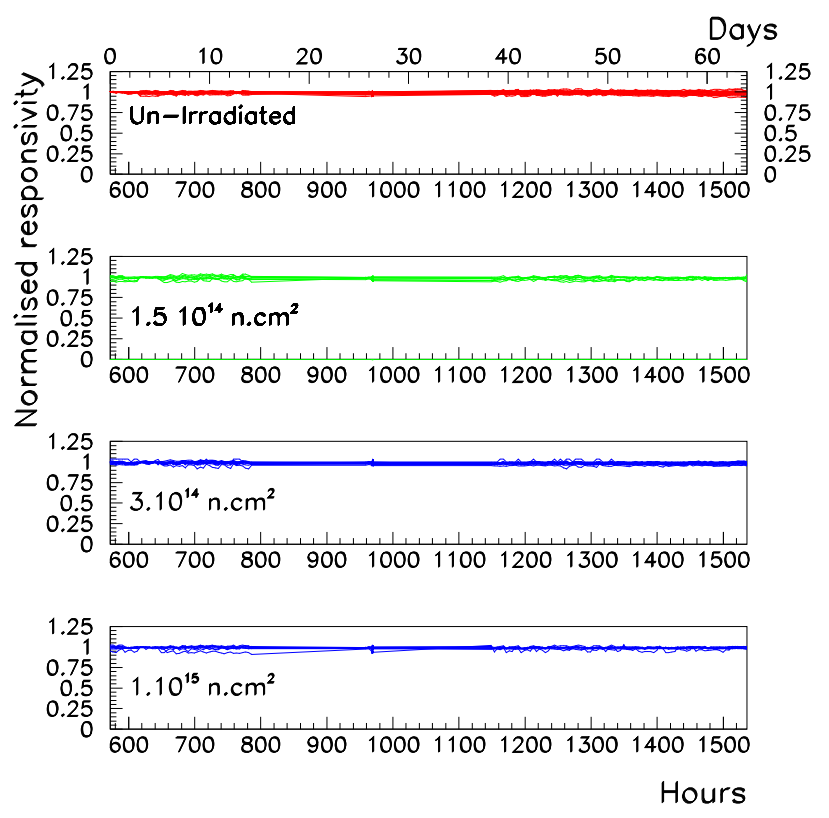

Fig. 12. Examples of the signals from different photodiodes as a function of time.

Better control of production processes than in the past and consequent increased wear out failure time results in a roughly constant failure rate over a limited time span. We will consider that $\lambda(\mathrm{t})=\lambda$. Therefore we can define the reliability $\mathrm{R}(\mathrm{t})$ as the Poisson probability that a single device does not fail within a time $\mathrm{t}$ : 


$$
R(t)=e^{-\lambda t}
$$

None of the PIN diodes has failed or shown any significant deterioration during the accelerated ageing tests. The probability $\mathrm{P}$ of no failure in a sample of $\mathrm{N}$ devices within a time $t_{a}$ is given by:

$$
P=\left(R\left(t_{a}\right)\right)^{N}
$$

So for a certain level of confidence $\mathrm{C}$, we can derive an upper limit on $\lambda$ :

$$
\lambda \leq-\frac{\ln (1-C)}{N t_{a}}
$$

Table 3 gives the values of $\lambda$ and MTTF at $90 \%$ confidence level for the unirradiated and irradiated PIN diode samples and the estimated upper limit on the number of faulty PIN diodes after 10 years in the SCT detector. The duration of the measurement $t_{a}$ is $5679 \mathrm{hr}$. An acceleration factor $A F=191$ was calculated using an activation energy of $0.6 \mathrm{eV}$ and conservatively taking the operational temperature $T_{S C T}$ to be $-7^{\circ} \mathrm{C}$. A further factor of 3 has been included to allow for the fact that the experiment will only operate for $1 / 3$ of the time. These are the conditions expected for the 2112 SCT detector modules in the central (barrel) region. If the 1976 modules in the forward region are operated at $0{ }^{\circ} \mathrm{C}$, the effective time is reduced by a factor of two and the failures after 10 years are approximately doubled.

Table 3

Lifetime lower limits for different PIN diode samples at $90 \%$ confidence level.

\begin{tabular}{|l|c|c|c|c|}
\hline $\begin{array}{l}\text { Fluence } \\
(1 \mathrm{MeV}) \\
\left(\mathrm{n} \mathrm{cm}^{-2}\right)\end{array}$ & $\begin{array}{c}\text { No. devices } \\
\text { tested }\end{array}$ & $\lambda$ & $M T T F\left(T_{S C T}\right)$ & $\begin{array}{c}\text { Max. no. of failures } \\
\text { in 2112 SCT barrel } \\
\text { devices after 10 years }\end{array}$ \\
\hline 0 & $23^{*}$ & $2.68 \times 10^{-4}$ & 3722 & 6 \\
$1.5 \times 10^{14}$ & 24 & $2.57 \times 10^{-4}$ & 3884 & 5 \\
$3 \times 10^{14}$ & 24 & $2.57 \times 10^{-4}$ & 3884 & 5 \\
$1 \times 10^{15}$ & 24 & $2.57 \times 10^{-4}$ & 3884 & 5 \\
\hline
\end{tabular}




\section{Conclusion}

The radiation hardness of epitaxial silicon PIN photodiodes has been tested up to an equivalent $1 \mathrm{MeV}$ neutron fluence of $10^{15} \mathrm{~cm}^{-2}$. The dark current reaches a maximum of less than $100 \mathrm{nA}$ which is comfortably below the typical operating photocurrent of $100 \mu \mathrm{A}$. There is a decrease in responsivity to about $70 \%$ of the unirradiated value which remains constant up to the maximun fluences tested. With a reverse bias of $5 \mathrm{~V}$, the average responsivity is $0.34 \mathrm{~A} / \mathrm{W}$ and the rise and fall times are less than $1 \mathrm{~ns}$ for fluences up to $3 \times 10^{14} \mathrm{n} \mathrm{cm}^{-2}$, which fully satisfies the requirements of the SCT detector. At $10^{15} \mathrm{n} \mathrm{cm}^{-2}$, a bias of $8 \mathrm{~V}$ is needed to achieve the same responsivity performance. The mean time to failure of the photodiodes is estimated to be greater than 3884 years with $90 \%$ confidence which would give an upper limit on the number of failures of 5 (out of 2112) after 10 years operation in the SCT barrel. The corresponding numbers for the SCT forward detector are 10 out of 1976. In both cases the expected failures are less than $1 \%$ which would be acceptable. In practice the effect would be even smaller because the readout scheme has a redundancy feature that allows the TTC signals to be taken from a neighbouring module in the event that any component of a module's TTC link fails. The photodiodes are also suitable for use at the higher radiation levels expected in the pixel detector.

\section{Acknowledgements}

We would like to acknowledge the assistance of Mike Edwards and Derrick Hill of the Rutherford Appleton Laboratory for the ISIS facility work, Dennis Grant, Roger Harris and Ian McGill at Birmingham for technical support, and Anne Fontaine at CERN for dosimetry services. We are indebted to Dr Jon Hall of Marconi for many helpful discussions about the performance of the PIN diodes, and to the Berne particle physics group for the use of the scanning machine. Financial support from the UK Particle Physics and Astronomy Research Council is gratefully acknowledged.

\section{References}

[1] D.G.Charlton et al., System Tests of Radiation Hard Optical Links for the ATLAS Semiconductor Tracker, accepted for publication in Nucl. Inst. and Meth. A (1999).

[2] ATLAS Inner Detector Technical Design Reports, CERN/LHCC/97-16/17, April 1997. 
[3] G.Battistoni, A.Ferrari and P.R.Sala, Background calculations for the ATLAS detector and hall, ATLAS Internal Note, ATL-GEN-94-010, October 1994.

[4] J.D.Dowell et al., Irradiation tests of photodiodes for the ATLAS SCT readout, Nucl. Inst. and Meth. A424 (1999) 483.

[5] T.F.Luera et al., Neutron Damage Equivalence for Silicon, Silicon Dioxide and Gallium Arsenide, IEEE Trans. Nucl. Sci. NS-34 (1987) 1557.

[6] A.van Ginneken, Non-ionizing Energy Deposition in Silicon for Radiation Damage Studies, Fermilab report FN-522 (1989).

[7] A.M.Ougouag, Differential Displacement Kerma Cross Section for Neutron Interactions in Si and GaAs, IEEE Trans. Nucl. Sci. NS-37 (1990) 2219.

[8] M.Huhtinen and P.A.Aarnio, Pion Induced Displacement Damage in Silicon Devices, Nucl. Inst and Meth. A335 (1993) 580.

[9] D.Pitzl et al., Type inversion in silicon detectors, Nucl. Inst. and Meth. A311 (1992) 98.

[10] J.Beringer et al., Radiation Hardness and Life Time Studies of LEDs and VCSELs for the Optical Readout of the ATLAS SCT, accepted for publication in Nucl. Inst. and Meth. A (1999).

[11] M.Edwards and D.R.Perry, The ISIS Radiation Hardness Test Facility, RAL90-065, August 1990.

[12] RD48 Collaboration: Status Report, CERN/LHCC/98-39, 1998.

[13] E.A.Weiss et al., Reliability Evaluation and Prediction for Silicon Photodetectors, IEEE Trans. Reliability, 37 (1988) 14-23.

[14] M.Ohring, Reliability and Failure of Electronic Materials and Devices, Academic Press, 1998. 